\title{
Health Care Assistants in Primary Care Depression Management: Role Perception, Burdening Factors, and Disease Conception
}

\author{
Jochen Gensichen, MD, MA, MPH ${ }^{1,2}$ \\ Cornelia Jaeger ${ }^{2}$ \\ Monika Peitz, $\mathrm{PbD}$ \\ Marion Torge, $M D^{2}$ \\ Corina Gütblin, $P b D^{2}$ \\ Karola Mergenthal \\ Vera Kleppel, ScD ${ }^{2}$ \\ Ferdinand M. Gerlach, MD, MPH \\ Juliana J. Petersen, $M D^{2}$ \\ 'Institute for General Practice, University \\ Hospital Jena, Friedrich-Schiller-University, \\ Jena, Germany \\ ${ }^{2}$ Institute for General Practice, Goethe- \\ University Frankfurt am Main, Germany
}

\begin{abstract}
PURPOSE In primary care, the involvement of health care assistants (HCAs) in clinical depression management is an innovative approach. Little is known, however, about how HCAs experience their new tasks. We wanted to describe the perceptions and experiences of HCAs who provided case management to patients with depression in small primary care practices.

METHODS This qualitative study was nested in the Primary Care Monitoring for Depressive Patients Trial on case management in Germany. We used a semistructured instrument to interview 26 HCAs and undertook content analysis. We focussed on 3 key aspects: role perception, burdening factors, and disease conception.

RESULTS Most HCAs said their new role provided them with personal and professional enrichment, and they were interested in improving patient-communication skills. They saw their major function as interacting with the patient and considered support for the family physician to be of less importance. Even so, some saw their role as a communication facilitator between family physician and patient. Burdening factors implementing the new tasks were the increased workload, the work environment, and difficulties interacting with depressed patients. HCAs' disease conception of depression was heterogeneous. After 1 year HCAs believed they were sufficiently familiar with their duties as case managers in depression management.
\end{abstract}

CONCLUSION HCAs were willing to extend their professional responsibilities from administrative work to more patient-centred work. Even if HCAs perform only monitoring tasks within the case management concept, the resulting workload is a limiting factor.

Ann Fam Med 2009;7:513-519. doi:10.1370/afm.1037

\section{INTRODUCTION}

AC Annals Journal Club selection; see inside back cover or http://www. annfammed.org/AJC/.

Conflicts of interest: none reported

CORRESPONDING AUTHOR

Jochen Gensichen, MD, MA, MPH

Institute for General Practice

University Hospital Jena

Friedrich-Schiller-University

Bachstr. 18, D- 07740

Jena, Germany

jochen.gensichen@med.uni-jena.de
$\Gamma$

ealth care assistants (HCAs) are providing an increasing part of primary health care services. Reports of clinical care provided by

HCAs in primary care depression management are available from the United States, the United Kingdom, the Netherlands ${ }^{1-5}$ and Germany. ${ }^{6}$ HCAs receive less training in patient care than do medical assistants or nurses, and they perform simple tasks according to defined protocols and procedures that require no clinical judgments or responsibility. They are accountable to a medical professional, ie, the family physician. ${ }^{7}$ The required qualifications for HCAs in Germany are graduation from high school and 3 years of vocational (on-the-job) training that includes a onehalf day of school per week. Their major tasks are focused on administration (as receptionists). HCAs always work under the supervision of a physician, who may delegate simple tasks, such as measuring blood pressure. ${ }^{8}$ In general, patients appreciate contact with nonprofessional or semi- 
professional staff, such as HCAs, because these staff have more time than the family physicians to listen the patients. ${ }^{9,10}$ Family physicians welcome the active role of the HCAs in clinical care when they have comprehensive knowledge of medications and sufficient experience in patient counseling or professional experience in mental health services. ${ }^{11,12}$ Major stress factors for HCAs and receptionists are difficult patients, patients' demands, and work pressure. ${ }^{13}$ Nurses, practice assistants, and HCAs complain of the psychological burden of providing mental health services in depression care. To avoid exhaustion, they prefer to work part-time. ${ }^{5}$ Reports of HCAs working in innovative depression care programs are still rare. ${ }^{14}$

This qualitative study was undertaken to provide perspective and information regarding the opportunities and limitations of HCAs working in primary care depression care.

\section{METHODS}

This qualitative study was nested in an intervention trial on depression case management (Primary Care Monitoring for Depressive Patients Trial, or PRoMPT). The trial examined the effect of case management for patients with major depression compared with usual care in small primary care practices. ${ }^{6}$ A practice-based HCA monitored patients by means of a monthly telephone call using the Depression Monitoring List to assess patients' depression symptoms and medication adherence. ${ }^{15}$ Results were reported to the family physician and, if necessary, discussed by the family physician and the HCA.

HCAs were not given specific orders how to integrate patient monitoring into their daily work. Their average caseload was 8 patients and ranged from 3 to 16 patients, and the case management responsibilities resulted in additional monitoring and administration duties. HCAs were trained in the basics of clinical depression and were taught communication skills using interactive methods, such as role playing and case studies. Each practice received a small financial compensation, which the family physician could divide up at will. The HCAs received no further payment. The study protocol was approved by the institutional review board. ${ }^{15,16}$ We are reporting our results in line with the standards for reporting qualitative research. ${ }^{17,18}$

\section{Study Participants and Sites}

We interviewed 26 of the 35 HCAs enrolled in the PRoMPT study, all of whom worked at different primary health care practices in Germany. All participants were invited to take part in the interviews by telephone. Five HCAs refused to be audio-recorded during the interview, and 4 refused to participate because of time constraints. The HCAs' sociodemographic characteristics are displayed in Table 1. The great majority had finished their 3-year professional training, but only a few had completed additional training or had any experience in caring for mental health patients. Most HCAs had had considerable work experience (mean, 16.5 years), typically at the same primary care practice. There were no substantial differences between responders (74\%) and nonresponders (26\%) in terms of relevant characteristics.

\section{Data Collection}

We developed a semistructured questionnaire based on a literature search and guided by the Chronic Care Model as our theoretical concept. ${ }^{19,20}$ This model advocates "productive interaction" between an "activated patient" and a "proactive practice team," with the aim of improving the clinical outcomes of patients with chronic diseases by emphasizing the importance of support for the patient by the entire practice team. The questionnaire was pretested, discussed, and refined in 2 focus group interviews: 1 with HCAs working as case managers $(\mathrm{n}=11)$, and 1 with HCAs

\section{Table 1. Sociodemographic Characteristics of Health Care Assistants}

\begin{tabular}{lcc}
\hline Characteristic & $\begin{array}{c}\text { HCA } \\
\text { Interviewed }\end{array}$ & $\begin{array}{c}\text { HCA } \\
\text { Not Interviewed }\end{array}$ \\
\hline Age, mean y & 39.5 & 44.1 \\
Female, \% & 100.0 & 100.0 \\
Family status, \% & & \\
Not married & 57.6 & 37.5 \\
Married, living together & 38.46 & 62.5 \\
Not specified & 3.85 & 0.0 \\
Graduation, \% & 11.55 & 12.5 \\
Higher than secondary school & 84.62 & 87.5 \\
Secondary modern school & 3.8 & 0.0 \\
Not specified & 88.4 & 75.0 \\
HCA with completed apprenticeship, \% & 10.4 & 8.7 \\
Time working in the current practice, mean y & 15.3 & 37.5 \\
Experience with psychiatric patients, \% & 3.1 & 3.8 \\
HCAs working in the practice, mean No. & & \\
Weekly hours of work, \% & 23.1 & 50.0 \\
Full time & 65.3 & 50.0 \\
Part time & 11.4 & 0.0 \\
Not specified & & \\
\hline HCA = health care assistants. & & \\
\hline
\end{tabular}


not working as case managers $(n=9)$. None was part of the following qualitative study.

The face-to-face interviews reported here were carried out in the general practice during the final visit of the study team at the end of the PRoMPT study after 1 year of experience. The interviews, which ranged from 23 to 60 minutes and lasted an average of 39 minutes, were undertaken by 2 authors (C.J. and V.K.) who had been trained and were experienced in conducting interviews. All interviews were audio-recorded and transcribed to written manuscripts.

\section{Data Analysis}

We conducted a computer-assisted content analysis according to Mayring ${ }^{21}$ using ATLAS.ti software, version 5.2 (ATLAS.ti, GmbH, Berlin, Germany). The complete manuscripts were independently read by 2 authors (J.G. and C.J.), both of whom then performed open coding for 7 (27\%) interviews and summarized the codes into categories. Categories were discussed until mutual agreement was reached. One researcher (C.J.) analyzed all the material using these categories, and categories were revised or expanded, if necessary. Three key aspects emerged: role perception, burdening factors, and disease conception. The final matching of categories and key aspects, as well as their validity, were discussed by a research team consisting of medical doctors, a psychologist, and PhD students, and agreement was reached when opinions differed.

\section{RESULTS}

\section{Role Perception}

Feelings about the new tasks and the roles described by almost all HCA interviewees fell into 2 categories. First, the HCAs emphasized a strong relationship with the study patients, stating that they had become a patient confidant and the person of first contact in the practice. They reported that before the study, patients had mainly addressed them on administrative issues, whereas now patients disclosed additional personal information.

They [patients] digressed from the questions to private topics. The question is just a kind of trigger and then...they started to tell me all the things they were really worried about (HCA 02).

Second, the HCAs believed the major aim of case management was to develop a "strong relationship" and to offer "additional conversation" to the patient. They assumed that the most important benefits for patients stem from increased attention, including more time to talk about problems, and the perception that someone cares about their problems:
One patient, she was...really upset and started crying like mad and then she thanked me afterwards; it had been such a great help that she could get it all off her chest. Just by listening I was able to help her (HCA 23).

Most HCAs perceived their new role as being personally and professionally enriching. They appreciated the "better insight" into medical issues and the personal satisfaction to be gained by "helping people" and receiving positive feedback from the patient. They believed these tasks were more consistent with their original reason for becoming an HCA:

Yes, it's really a lot of fun. To get away from the usual practice work-all the "what are the findings, how was my blood sample, how is this, and what was that?" Then I ask myself why I became an HCA in the first place. To spend the whole day bending down, opening boxes, and looking for patients' files? It really makes a big change, it's a challenge, and it's fun to think you've actually been of use (HCA 05).

Some HCAs described that they tried to listen to the patients and to provide them with additional advice and support:

Let's put it like this. I understand it, and sometimes I try to...get involved myself, so that they might somehow get or find a way of pulling themselves out of it (HCA 10).

Some emphasized that they attached great importance to keeping "a certain distance" between themselves and the patient. On the other hand, there were HCAs who described a very "intimate and empathic" way of dealing with the patients, such as, for example, being on first-name terms or tolerating direct body contact, such as hugging or cheek kissing.

HCAs described their tasks as focused mainly on direct communication with the patients. Providing the doctors with information on symptoms and medication was seen as less important. Only a few HCAs mentioned the relevance of the information for the doctor's treatment planning:

I would say it was rather more like looking after the patient. Supporting the doctor is good as well, of course, for them anyway. It makes the job a bit easier when they know in advance what the problem is and what they have said, but most of all it's the patients who are helped (HCA 28).

Saving time was reported as a benefit for the physician because patients often contacted the HCA before contacting their physician. Additionally, some HCAs believed they acted as a communication facilitator between the physician and the patients. Although HCAs perceived the doctor's role in case management to be of minor importance, most were keen to inform the doctor about the patient's status and did so in a regular and timely manner. 


\section{Burdening and Relieving Factors}

Factors that either aggravated or reduced the workload regularly emerged from the interviews.

\section{Time and Organization}

Many HCAs believed that it was difficult to integrate case management into their daily work, either because of insufficient time or unexpected patient-related factors, such as missed appointments or emotional responses that needed attention. Some HCAs even started to make telephone calls to patients during their free time:

The [conversations] get longer and longer in this study. In the beginning we started [with] 5 or 7 minutes, and now it's not rare to have a conversation that lasts half an hour (HCA 06).

In some cases, these time limitations were aggravated by colleagues and bosses' lack of understanding:

Not even the HCAs could accept that we weren't to be disturbed at such moments. That is bad. There were even situations when the boss started hammering on the door and saying, "You are going to have to come and help [at the front desk]" (HCA 05).

On the other hand being given the time by the doctor was considered very helpful.

[My boss]... knew what I was doing and accepted the situation and gave me the necessary time to do it (HCA 15).

\section{Interaction With Depressed Patients}

Many HCAs found contact with depressed patients to be difficult, applying such descriptors as "exhausting," "sensitive," "reserved," "unpredictable," "bad-tempered," "complaining," "annoying," "egomaniac," and "demanding." The HCAs believed that dealing with depressed patients required special skills of attentiveness, sensitivity, and caution. Some reported having difficulties when they were confronted with patients who became emotional. In these cases, HCAs felt insecure in their role as case managers:

[It was] pretty tough. Because you don't know exactly how to react. I am neither a doctor nor a psychotherapist nor a best friend (HCA 05).

In addition, some HCAs were anxious about their own "mental well-being," when confronted with so much misfortune and desperate life situations:

Then she goes and tells me that she's been beaten up by her husband. When you know such things, you carry them around with you. I've got a kind of neurotic need to help and imagine I have to save the world (laughs). So of course I had to take it all back home with me (HCA 06).

Referring to the depression-monitoring instrument, the interviewees mentioned 2 difficulties: asking about suicidal ideation, and asking whether the patient felt a "burden for others."

For me, the question was always unpleasant, every time. "Are you considering suicide?" That really is something intimate, and it takes my breath away to have to... ask directly (HCA 23).

HCAs were afraid not "to react appropriately" and fast enough in case a patient did agree:

Yes, that someone might really say, "I feel so bad," or "Me? Me? Yes, red alert, terrible." That you have to react quickly and then.... And the question how to deal with the situation with the patient (HCA 21).

HCAs believed that asking whether interviewees felt themselves to be a burden went beyond their responsibilities:

The question could never be a simple one. Some kind of explanation always had to be given. You also noticed how the train of thought was triggered, how they were thinking-Am I a failure, or am I not a failure? I really thought... this is pretty tough. You may be able to do it when a doctor or a psychotherapist is present when you ask the question, but when you touch on such a subject in such a trivial, in inverted commas trivial, question-and-answer situation, I think it's pretty serious (HCA 26).

\section{Collaboration With the Doctor}

Another factor influencing the perceived degree of stress was the extent to which the family physician got involved. Even though most interviewees emphasized what was generally good collaboration with their bosses, many found it difficult to regard case management as teamwork:

Cooperation? No, I couldn't really say that-I did my job, and she did hers (HCA 15).

Substantial stress was also reported when HCAs believed that the doctors put too little effort into supporting them.

To be honest, sometimes he didn't go into the whole problem of depression in enough depth. I would sometimes have preferred it if he had paid a little more attention to my needs. Basically, he just did what he is supposed to do; he just relied on me to indicate when it was time for him to get involved. But he never tried to find time to go into it all in more depth; never said I want to see how things are. He had to rely on me to watch out for them, for the patients. Basically, you can almost say that responsibility was handed over to me a bit (HCA 26).

The presence of the doctor, however, was also perceived by the HCAs as relief from the burden of responsibility. Because the physicians were requested to always be available either by telephone or in person, 
HCAs always knew that in acute situations the doctors would take charge.

If I had had the impression that the situation was really getting out of hand, that something really needed to be done, then I would have sent for the doctor. That was always in the back of my mind (HCA 20).

\section{More Relieving Factors}

Experience gained during the 1-year study helped the HCAs deal more effectively with their case management work. Furthermore, "a certain age and maturity" was considered necessary to maintain the required inner strength:

You always find out things about people's personal lives, and I have got so used to it that, just as I never bring any of my personal life into the practice, I try not to take anything out either (HCA 10).

The "relationship with the patients" was a frequently named aspect for reducing the strain, but in differing ways. Although some HCAs pointed out that "distance to the patients" helped them to deal with "certain situations," others regarded a long-standing personal acquaintance with patients as helpful:

I've known the patient for at least 15 years, so I know his situation well. I'd probably have been shocked if it had been anyone else, but I wasn't because it was him (HCA 15).

\section{Disease Conception}

We asked the HCAs about their knowledge of the cause, treatment, and prognosis of depression. Most interviewees said their attitude toward the disease had changed during the year as a case manager-they were now "more willing to accept" depression to be "an actual disease" and found it easier to act in "an understanding manner." Thus, their awareness of depression symptoms, even among patients not included in the study, had increased:

Depression used to be something where you just thought they shouldn't make such a fuss. But now I view things differently. It's an illness for me now and, yeah, you're just more understanding. Actually, I think that's been a really important thing to have gone through. For me personally, yeah, the fact that I see things completely differently now, that I've broadened my horizons over the last year (HCA 06).

Even so, medical knowledge about depression proved rudimentary for most interviewees. Being asked about the causes of the disease, "difficult life situations" and "social change" had top priority, whereas it was not clear to them that the affective disorder itself causes a lack of drive and a pessimistic view of life. In some cases, their incomplete understanding of the disease increased the strain on HCAs by enhancing their feeling of being unable to help:

When the social welfare office cuts back on their payments and they only have $€ 50$ left to live on..., I mean, you just don't know what to say. You just ask yourself how this...person is supposed to ever come out of his or her depression (HCA 08).

On being asked what was beneficial for depressed patients, the HCAs put much emphasis on the "empathic treatment" of the patient in the form of listening, being patient, and understanding. Of the professional treatment options, they clearly favored conversational therapy, being very dismissive of pharmacotherapy, which they considered to be appropriate only if combined with psychotherapy or if used temporarily:

I think they just need someone to talk to, a chance to speak openly to someone they trust, a chance to really let it all out. Just to know someone is there for them that they can talk to, to whom they can turn (HCA 24).

Concerning the depression prognosis, the interviewees had a rather pessimistic view, even though they tended to distinguish between levels of severity. Many believed symptoms can merely be relieved and thus that the disease is not curable.

\section{DISCUSSION}

This study is the first to examine how HCAs without any specialist mental health training experience a new role as case managers for depressed patients in primary care clinics. Current literature suggests that medical knowledge, counseling skills, and professional experience are perceived by physicians as being crucial for HCAs when dealing with mentally ill patients. ${ }^{22-24}$ Patients, on the other hand, like to talk to someone who is semiprofessional and often has more time to listen. ${ }^{5,9,10}$ Our results show that HCAs are willing to extend their professional responsibilities from administrative work to include direct contact with patients. When dealing with mental diseases, however, the strain resulting from interaction with the patients should not be underestimated. In our study, we found that misconceptions about depression may reduce HCAs capacity to deal with the tasks of depression case management. Hence, we propose attaching substantial importance to the training of future case managers (eg, clinical knowledge and counseling principles). In addition, HCAs' tasks should be clearly defined, and strategies to prevent overburdening should be taught. Even if HCAs only monitor symptoms in patients, the 
resulting increased workload stemming from regular telephone calls and structured feedback to the physician should be taken into consideration. Financial compensation should also be required for additional working hours when implementing case management on a large scale. Moreover, case management should not be understood as a 1-way shift of tasks, workload, and clinical responsibilities from the physician to HCA. ${ }^{25}$ In line with other research, ${ }^{26-28}$ the tasks of the physician should be clearly defined to prevent HCAs from feeling too responsible. HCAs perceived the shared responsibility to be both a burden and a relief. Although it appeared that case management shifted responsibility in patient care to the HCAs, the final clinical responsibility was with the physician. Because the case management approach included clear-cut information on when the physician should get involved, the shared responsibility of case management was also perceived as providing relief.

The strength of this study is that it is the first to examine the implementation of case management in small-scale primary practices, ${ }^{6}$ as well as the perception of the HCA in this specific setting. It seems that the focus on a single perspective of this topic - the perspective of the HCAs_-is both a strength and a weakness. It is a strength because the study provides an in-depth understanding of this perspective. It is a weakness because there are other important perspectives to consider, such as, for example, patients' and physicians' perspectives, and those perspectives have not yet been examined.

A limitation of the study is that (for administrative reasons and although generally recommended) participants were not asked to confirm the interview findings. ${ }^{16}$

In summary, it can be said that HCAs can fulfill the tasks of depression case management in general practice and that they find these tasks personally enriching. Several burdening and enabling factors for HCAs adapting to this new role should be taken into consideration when implementing case management.

To read or post commentaries in response to this article, see it online at http://www.annfammed.org/cgi/content/full/7/6/513.

Submitted September 22, 2008; submitted, revised May 6, 2009; accepted May 19, 2009.

Key words: Health care assistants; allied health personnel; depressive disorder; primary health care

Funding support: Support for the Primary Care Monitoring for depressive Patients Trial (PRoMPT) came from the German Ministry of Education and Research (DLR 01GK0302).

Disclaimer: None of the funding agencies had any role in the design of the study, analysis of data, or preparation of the manuscript.
Acknowledgment: This study has been awarded by the Lothar-BeyerAward 2008/German Primary Care Research Award 2008.

\section{References}

1. Unützer J, Katon W, Callahan CM, et al. IMPACT Investigators. Improving mood-promoting access to collaborative treatment. Collaborative care management of late-life depression in the primary care setting: a randomized controlled trial. JAMA. 2002;288(22): 2836-2845.

2. Richards DA, Lovell K, Gilbody S, et al. Collaborative care for depression in UK primary care: a randomized controlled trial. Psychol Med. 2006;38(2):279-287.

3. Chew-Graham CA, Lovell K, Roberts C, et al. A randomised controlled trial to test the feasibility of a collaborative care model for the management of depression in older people. Br J Gen Pract. 2007;57(538):364-370.

4. Dietrich AJ, Oxman TE, Williams JW Jr, et al. Re-engineering systems for the treatment of depression in primary care: cluster randomised controlled trial. BMJ. 2004;329(7466):602.

5. Symons L, Tylee A, Mann A, et al. Improving access to depression care: descriptive report of a multidisciplinary primary care pilot service. Br J Gen Pract. 2004;54(506):679-683.

6. Gensichen J, von Korff M, Peitz M, et al. Case management for depression by health care assistants in small promary care practices-a cluster randomized trial. Ann Intern Med. 2009;151(6): 369-380.

7. Bosley S, Dale J. Healthcare assistants in general practice: practical and conceptual issues of skill-mix change. Br J Gen Pract. 2008;58(547):118-124.

8. Brant $\mathrm{C}$, Leydon GM. The role of the health-care assistant in general practice. Br J Nurs. 2009;18(15):926; 928-390; 932-933.

9. Crosland A, Kai J. 'They think they can talk to nurses': practice nurses' views of their roles in caring for mental health problems. BrJ Gen Pract. 1998;48(432):1383-1386.

10. Backenstrass M, Joest K, Rosemann T, Szecsenyi J. The care of patients with subthreshold depression in primary care: is it all that bad? A qualitative study on the views of general practitioners and patients. BMC Health Serv Res. 2007;7:190.

11. Richards DA, Lankshear AJ, Fletcher J, et al. Developing a U.K. protocol for collaborative care: a qualitative study. Gen Hosp Psychiatry. 2006;28(4):296-305.

12. Rosemann T, Joest K, Körner T, Schaefert R, Heiderhoff M, Szecsenyi J. How can the practice nurse be more involved in the care of the chronically ill? The perspectives of GPs, patients and practice nurses. BMC Fam Pract. 2006;7:14.

13. Eisner M, Britten N. What do general practice receptionists think and feel about their work? Br J Gen Pract. 1999;49(439):103-106.

14. Thielke $S$, Vannoy $S$, Unützer J. Integrating mental health and primary care. Prim Care. 2007;34(3):571-592, vii.

15. Gensichen J, Peitz M, Torge M, et al. The "Depression Monitoring list" (DeMoL) with integrated PHQ-D-Rationale and design of a tool for the case management for depression in primary care [in German]. Z Arztl Fortbild Qualitatssich. 2006;100(5):375-382.

16. Gensichen J, Torge M, Peitz M, et al. Case management for the treatment of patients with major depression in general practicesrationale, design and conduct of a cluster randomized controlled trial-PRoMPT (PRimary care Monitoring for depressive Patient's Trial) [ISRCTN66386086] - study protocol. BMC Public Health. 2005;5:101.

17. Tong A, Sainsbury P, Craig J. Consolidated criteria for reporting qualitative research (COREQ): a 32-item checklist for interviews and focus groups. Int J Qual Health Care. 2007;19(6):349-357. 
18. Elliott R, Fischer CT, Rennie DL. Evolving guidelines for publication of qualitative research studies in psychology and related fields. $\mathrm{Br}$ Clin Psychol. 1999;38(Pt 3):215-229.

19. Wagner EH, Austin BT, Von Korff M. Improving outcomes in chronic illness. Manag Care Q. 1996;4(2):12-25.

20. Wagner EH, Austin BT, Davis C, Hindmarsh M, Schaefer J, Bonomi A. Improving chronic illness care: translating evidence into action. Health Aff (Millwood). 2001;20(6):64-78.

21. Mayring P. Qualitative Inhaltsanalyse. [Content Analysis]. UTB: Berlin; 2007.

22. Katon W, Von Korff M, Lin E, et al. Stepped collaborative care for primary care patients with persistent symptoms of depression: a randomized trial. Arch Gen Psychiatry. 1999;56(12):1109-1115.

23. Nutting PA, Gallagher KM, Riley K, White S, Dietrich AJ, Dickinson WP. Implementing a depression improvement intervention in five health care organizations: experience from the RESPECT-Depression trial. Adm Policy Ment Health. 2007;34(2):127-137.
24. Bowman S, Bray K, Leaver G, Pilcher T, Plowright C, Stewart L; British Association of Critical Care Nurses. Health care assistants' role, function and development: results of a national survey. Nurs Crit Care. 2003;8(4):141-148.

25. Ramprogus $V, O^{\prime}$ Brien $D$. The case for the formal education of HCAs. Nurs Times. 2002;98(27):37-38.

26. Chang AM, Lam L. Can health care assistants replace student nurses? J Adv Nurs. 1998;27(2):399-405.

27. Cunningham R. Professionalism reconsidered: physician payment in a small-practice environment. Health Aff (Millwood). 2004;23(6):36-47.

28. Cohen DJ, Crabtree BF. Evaluation criteria for qualitative research in health care: controversies and recommendations. Fam Med. 2008;6(4):331-339.

\section{CHANCE-OF-ADDRESS FORM FAMILY MEDICINE}

Please complete this form and mail to the following address or fax to Annals Circulation at 913-906-6080:

Annals of Family Medicine, Circulation Department, 11400 Tomahawk Creek Pkwy, Leawood, KS 66211-2680

Check if member of sponsoring organization:

$\begin{array}{ll}\square \text { AAFP } & \square \text { ABFM } \square \text { STFM } \square \text { ADFM } \\ \square \text { AFMRD } \square \text { NAPCRG } \square \text { CFPC }\end{array}$

ID number from label on your journal cover

OLD Information (Please print.)

Name

Company (if applicable)

Address (Street plus Apt or Ste)

\begin{tabular}{ll}
\hline City & State \\
\hline Country & Postal Code (9-digit ZIP for US) \\
\hline Telephone & Fax \\
\hline E-Mail &
\end{tabular}

NEW Information (Please print.)

\begin{tabular}{ll}
\hline Name \\
\hline Company (if applicable) \\
\hline Address (Street plus Apt or Ste) & \\
\hline City & Postal Code (9-digit ZIP for US) \\
\hline Country & \\
\hline Telephone & \\
\hline E-Mail &
\end{tabular}

